\title{
Mortalidade perinatal e neonatal no Hospital de Clínicas de Porto Alegre
}

\author{
E. MIURA, L.H. FAILACE, H. FIORI
}

Unidade de Neonatol ogia do Hospital de Clínicas de Porto Alegre, Serviço de Pediatria. Faculdade de M edicina da Universidade F ederal do Rio Grande do Sul, Porto Alegre, RS.

\begin{abstract}
RESUMO - Овj etivo. Análise epidemiológica da mortalidade neonatal e perinatal de $\mathbf{2 0 . 2 8 0}$ crianças nascidas vivas com $500 \mathrm{~g}$ ou mais e 374 natimortos ocorridas no Hospital de Clínicas de Porto Alegre, no período de 1984 a 1990.

Proposta. Comparar dois períodos: A (1984-1987) com B (1988-1990), estabelecendo as mudanças ocorridas.

Métodos. É um estudo retrospectivo de revisão dos registros de nascimentos do centro obstétrico, internações e óbitos da unidade neonatal e mortes fetais e dos laudos de necrópsia.

Resultados. Faleceram 258 R N, com um coeficiente de mortalidade neonatal de 12,7 por mil. A taxa de natimortalidade foi de 18,4 por mil. $O$ coeficiente de mortalidade perinatal foi de $\mathbf{2 8 , 4}$ por mil. A incidência de baixo peso ao nascer $(<2.500 \mathrm{~g})$, de $11,2 \%$, e a de muito baixo peso ao nascer $(<1.500 \mathrm{~g})$ foi de $1,8 \%$. Este último grupo aumentou sua incidência de 1,5\%
\end{abstract}

\section{NTRODUÇÃO}

Em Porto Alegre, no ano de 1991, a mortalidade infantil foi de 13,8 por mil, sendo a mortalidade neonatal responsável por $58,1 \%$ dos óbitos. E mais da metade dos óbitos da mortalidade infantil tardia estão associados à prematuridade ${ }^{1}$. A análise dessas causas são usadas pelos profissionais de saúde no planejamento e tomada de decisões nos programas de saúde. $O$ estudo permanente das causas de morte fetal e neonatal de um hospital permite identificar as condições de saúde da população servida e a qualidade de assistência perinatal oferecida, facilitando as correções das distorções detectadas ${ }^{2-4}$. A classificação da mortalidade perinatal de Quebec, Canadá , é utilizada no H ospital de Clínicas de Porto Al egre (HCPA), a exemplo da mai oria dos serviços de perinatologia.

Este trabal ho analisou os coeficientes de mortalidade neonatal e perinatal durante sete anos, e comparou dois períodos: (A) 1984-1987 versus (B) 19881990, com o objetivo de comparar as diferenças nas enfermidades básicas causadoras de mortes intrauterinas e neonatais e tendências dos óbitos por grupos de peso.
(A) para $2,2 \%$ (B). As causas mais freqüentes de morte neonatal foram: a) infecção intra-uterina $(22,4 \%) ;$ b) doença de membrana hialina $(20,1 \%) ; c)$ malformações múltiplas (18,2\%); d) asfixia perinatal $(15,5 \%)$; e) infecções pós-natais específicas $(9,7 \%)$. As causas de morte fetal foram: a) asfixia (38,7\%); b) infecções intra-uterinas (9\%); c) toxemia $(8,2 \%)$; d) malformações múltiplas $(7,4 \%)$. No período $B$, foi significativo o aumento de óbitos por infecções pósnatais, OR $7(1,9-30,6)$ e por malformações congênitas, OR 1,6 (0,8-3,2). Não houve redução significativa na taxa de mortalidade em menores de $1.500 \mathrm{~g}$ OR 90 (61-118) em A para 54 (37-68) em B.

ConClusÃo. Não houve redução nas taxas de mortalidade neonatal, apesar dos avanços tecnológicos e na capacitação técnica.

UNITERMOS: Recém-nascido. Mortalidade perinatal. Mortalidade neonatal.

\section{MATERIAL E MÉTODOS}

Os dados foram obtidos por meio da revisão dos registros do centro obstétrico e da unidade neonatal do HCPA, bem como da revisão dos prontuários de todos os óbitos dos fetos e dos RN nesse período e dos laudos de necrópsia, quando disponíveis. Esses dados foram agrupados por faixas de peso de $500 \mathrm{~g}$, a partir de $500 \mathrm{~g}$. Foram calculados os seguintes coeficientes: natimortalidade ou mortalidade fetal, mortalidade neonatal precoce (até 7 dias), mortalidade neonatal tardia (8-28 dias), perinatal, que é a soma da natimortalidade e a mortalidade neonatal precoce. Foram comparadas as tendências de óbitos nos dois períodos nos RN de baixo peso $(<2.500 \mathrm{~g})$ e de muito baixo peso $(<1.500 \mathrm{~g})$ por meio das razões de riscos de óbitos (OR=odds ratio), com intervalo de confiança $(\mathrm{Cl})$ de $95 \%$.

\section{RESULTADOS}

No período de 1ํ de janeiro de 1984 a 31 de dezembro de 1990, nasceram na maternidade do HCPA 20.280 crianças vivas com $500 \mathrm{~g}$ ou mais, e 374 


\begin{tabular}{|c|c|c|c|c|c|c|}
\hline $\begin{array}{c}\text { Peso } \\
\text { (g) }\end{array}$ & $\begin{array}{c}\text { № } \\
\text { nascidos } \\
\text { vivos }\end{array}$ & $\begin{array}{c}\text { № } \\
\text { partos }\end{array}$ & $\begin{array}{c}\text { № } \\
\text { óbitos } \\
\text { neonatais }\end{array}$ & $\begin{array}{c}\text { Mortalidade } \\
\text { neonatal } \\
\text { por mil }\end{array}$ & $\begin{array}{c}\text { № } \\
\text { óbitos } \\
\text { até } 7 \text { dias }\end{array}$ & $\begin{array}{c}\text { № } \\
\text { óbitos } \\
\text { 8-28 dias }\end{array}$ \\
\hline 500-999 & 143 & 138 & 102 & 713,3 & 81 & 21 \\
\hline $1.000-1.499$ & 227 & 217 & 40 & 176 & 32 & 8 \\
\hline $1.500-1.999$ & 517 & 487 & 41 & 79 & 30 & 11 \\
\hline $2.000-2.499$ & 1.401 & 1.324 & 18 & 12,8 & 16 & 2 \\
\hline $2.500-2.999$ & 4.241 & 4.164 & 24 & 5,6 & 17 & 7 \\
\hline $3.000-3.499$ & 7.699 & 7.666 & 16 & 2,07 & 12 & 4 \\
\hline 3.500-3.999 & 4.661 & 4.651 & 6 & 1,3 & 4 & 2 \\
\hline$>4.000$ & 1.357 & 1.357 & 2 & 1,4 & 2 & - \\
\hline Desconhecido & 34 & 34 & 9 & - & 9 & - \\
\hline Total & 20.280 & 20.038 & 258 & 12,7 & 203 & 55 \\
\hline
\end{tabular}

crianças nascidas mortas. A distribuição dos recémnascidos (RN) por faixas de peso de $500 \mathrm{~g}$ é mostrada na tabela 1. N essa mesma tabela são apresentados os índices de mortalidade neonatal, neonatal precoce e neonatal tardia, por faixas de peso.

\section{DISCUSSÃO}

Na tabela 1, os dados mostram que o coeficiente de mortalidade neonatal hospitalar do HCPA, no período de 1984 a 1990, foi de 12,7 por mil, a fetal, 16,8 por mil, e a perinatal, 28,4 por mil. O coeficiente de mortalidade neonatal é semelhante aos hospitais terciários de países industrializados, como os retirados do Women's College Hospital, de Toronto, no período de 1980-1984: 11,6 por mil7. Os dados de hospitais brasileiros disponíveis são os do Hospital Regional da Asa Sul, Brasília, que mostram, no período de 1985-1991, mortalidade perinatal de 35,4 por mil, mortalidade fetal de 19,6 por mil e mortalidade neonatal de 16 por milis. As estatísticas da Maternidade de Cachoeirinha, São Paulo, de 1982, revelaram taxa de neomortalidade de 17,2 por mil, sendo 600,3 por mil a mortalidade em menores de $1.500 \mathrm{~g}^{9}$. E sses resultados são comprometedores, pois revelam as más condições socioeconômicas da população e de assistência médica perinatal ${ }^{3,9}$.

A maioria dos bebês morre nos primeiros sete dias de vida, $84 \%$ do total, evidenciando a gravidade das enfermidades que acometem o recém-nascido, além de mostrar as más condições de nascimento?.

Os RN menores de $2.500 \mathrm{~g}$ (baixo peso) representaram $11,2 \%$ do total dos nascimentos, porém foram responsáveis por mais de $80 \%$ do total de óbitos; e os RN menores de $1.500 \mathrm{~g}$ (muito baixo peso) represen-
Tabela 2-Mortalidade neonatal em recém-nascidos de baixo peso e de muito baixo peso no Hospital de Clínicas de Porto Alegre

Peso ao nascer Nascidos 1984-1987 Nascidos 1988-1990 (g) vivos óbitos OR vivos Óbitos OR

$\begin{array}{lrrrrrr}500-1.499 & 155 & 67 & 90 & 215 & 75 & 54 \\ 1.500-2.499 & 1.152 & 37 & 39 & 1.136 & 25 & 24\end{array}$

Razão de riscos (OR) morte neonatal $<1.500 \mathrm{~g}(\mathrm{Cl} 95 \%)=90(61-118)$ vs. 54 (37-68) não-significativo.

OR morte neonatal 1.500-2.500g $(\mathrm{Cl} 95 \%)=39(24-61)$ vs. 24 (16-34) nãosignificativo.

taram somente $1,8 \%$ do total de nascimentos e foram responsáveis por $57 \%$ do total de óbitos. Este grupo ocupa mais de $80 \%$ dos leitos de UTI e tem custos extraordinariamente caros. Também nos centros regionais de terapia intensiva neonatal de países desenvolvidos tecnologicamente, os RN de baixo peso e, mais particularmente, os de muito baixo peso representam $85 \%$ da mortalidade neonatal. Portanto, a prematuridade é o fator de risco mais importante determinante das graves patologias responsáveis pela morbi-mortalidade neonatal ${ }^{6,10,11}$.

Observa-se aumento progressivo no número de nascimentos de menores de $1.500 \mathrm{~g}$ no HCPA e que hoje representam mais de $3 \%$ do total de nascimentos. Houve a aquisição de novos equipamentos na terapia intensiva, treinamento continuado do pessoal médico e de enfermagem. Entretanto, não houve redução significativa quanto às taxas de mortalidade. Apenas uma tendência na redução da mortalidade em menores de $1.500 \mathrm{~g}$, cuja razão de riscos (odds ratio) caiu de 90 (61-118) para 54 (37-68), com intervalo de confiança de $95 \%$. N os RN de moderado baixo peso (1.500-2.500), houve uma leve tendência 
Tabela 3 - Causas básicas de mortalidade neonatal no Hospital de Clínicas de Porto Alegre

\begin{tabular}{|c|c|c|c|c|c|}
\hline \multirow[t]{2}{*}{ Causas } & \multicolumn{2}{|c|}{$1984-1987$} & \multicolumn{2}{|c|}{$1988-1990$} & \multirow[t]{2}{*}{ OR } \\
\hline & $\begin{array}{l}\text { No de } \\
\text { casos }\end{array}$ & $\%$ & $\begin{array}{l}\text { No de } \\
\text { casos }\end{array}$ & $\%$ & \\
\hline Infecções agudas intra-uterinas & 32 & 27,1 & 26 & 18,4 & NS \\
\hline Doença da membrana hialina & 26 & 22,0 & 26 & 18,4 & NS \\
\hline Asfixia perinatal & 20 & 16,9 & 20 & 14,2 & NS \\
\hline Malformações múltiplas & 17 & 14,4 & 30 & 21,2 & $1,6(0,8-3,2)$ \\
\hline Infecções pós-natais & 3 & 2,5 & 22 & 15,6 & $7,0(1,9-30,6)$ \\
\hline Hemorragia intracraniana & 3 & 2,5 & 2 & 1,4 & NS \\
\hline Outros distúrbios respiratórios & 2 & 1,7 & 4 & 2,8 & NS \\
\hline Desnutrição fetal & 2 & 1,7 & - & - & NS \\
\hline Isoimunização Rh & 1 & 0,8 & 1 & 0,7 & NS \\
\hline Hidropsia fetal & 1 & 0,8 & - & - & NS \\
\hline Causa desconhecida & 11 & 9,3 & 10 & 7,1 & NS \\
\hline Total & 118 & 100,0 & 141 & 100,0 & \\
\hline
\end{tabular}

de redução com OR 39 (24-61) para 24 (16-34). Os dados da Carolina do N orte (EUA) (1980-1984) mostraram que o OR de óbito para os menores $1.500 \mathrm{~g}$ é semelhante ao do HCPA, ou seja, de 85, porém, nos RN de 1.500 a $2.500 \mathrm{~g}$, o OR é de somente $6^{2}$. Esses resultados revelam as deficiências no atendimento a gestante, feto e recém-nascido da equipe perinatal do HCPA, com prevalência de enfermi dades em fase avançada, como a doença da membrana hialina, asfixia e infecções ${ }^{3}$.

Recentemente, comparamos a sobrevida dos prematuros de muito baixo peso, no período de janeiro a junho de 1991, com os dados de um estudo multicêntrico de sete centros universitários norte-americanos, em que a sobrevida do HCPA foi semel hante - $71 \%$ versus $74 \%$ de sobrevida nos sete centros dos EUA. Houve diferença nos prematuros menores de $750 \mathrm{~g}$, em que a mortalidade no HCPA foi de $87 \%$ versus $45 \%$ (EUA) ${ }^{12}$. I sso mostra que ainda somos incapazes de dar uma assistência adequada para os RN menores de 750g.

Em nosso serviço, o número de natimortos de 16,8 por mil é exageradamente elevado, se comparado com a taxa de 6 por mil de Toronto e outros hospitais norte-americanos ${ }^{7,10,13}$. Novamente, é um reflexo do mau atendimento pré-natal, a má utilização dos serviços pré-natais pelas gestantes, o encaminhamento tardio das gestantes com sofrimento fetal, encaminhamento tardio das gravidezes de alto risco para os hospitais de referência9. As más condições socioeconômicas e culturais das pacientes também dificultam a eficiência do atendimento médi $\mathrm{CO}^{10,12}$.

A prematuridade está freqüentemente associada às causas básicas de mortalidade neonatal: as infecções agudas intra-uterinas (sepse precoce e tardia
Tabela 4 - Causas básicas de natimortalidade no Hospital de Clínicas de Porto Alegre

\begin{tabular}{|c|c|c|c|c|}
\hline \multirow[t]{2}{*}{ Causas básicas } & \multicolumn{2}{|c|}{$1984-1987$} & \multicolumn{2}{|c|}{$1988-1990$} \\
\hline & № casos & $\%$ & № casos & s $\%$ \\
\hline $\begin{array}{l}\text { Asfixia perinatal por } \\
\text { descolamento prematuro } \\
\text { de placenta, circular e } \\
\text { nó de cordão, outras } \\
\text { patologias de cordão, } \\
\text { infarto placentário, } \\
\text { anomalias de trabalho }\end{array}$ & & & & \\
\hline de parto & 66 & 37,2 & 79 & 40,1 \\
\hline Infecções intra-uterinas & 20 & 11,3 & 14 & 7,1 \\
\hline Toxemia & 10 & 5,6 & 21 & 10,6 \\
\hline Malformações múltiplas & 9 & 5,1 & 19 & 9,6 \\
\hline Sífilis & 5 & 2,8 & 1 & 0,5 \\
\hline Isoimunização Rh & 4 & 2,3 & 5 & 2,5 \\
\hline Outras doenças maternas & 5 & 2,8 & 4 & 2,0 \\
\hline Diabetes melito & 3 & 1,7 & 2 & 1,0 \\
\hline Listeriose & 1 & 0,6 & 2 & 1,0 \\
\hline Desconhecido & 54 & 29,9 & 50 & 25,4 \\
\hline Total & 177 & 100,0 & 197 & 100,0 \\
\hline
\end{tabular}

não-hospitalar) passaram a ser a primeira causa de morte neonatal no período $A$; o mesmo ocorreu com a doença de membrana hialina. As infecções pósnatais ou hospitalares passaram a ser a terceira causa de morte no período $B$, devido a maior sobrevida dos prematuros e maior vigilância no registro de infecções hospital ares ${ }^{3}$. As malformações graves tornaram-se a primeira causa de morte, à semeIhança dos hospitais $7,16,17$ em que nem sempre o fator prematuridade está presente. Essas doenças indicam aumento na transferência de gravidezes de risco para os hospitais terciários. Os dados de mortalidade neonatal, em 16 hospitais do Rio Grande do 
Sul com 307 óbitos (1984-1986), mostraram a seguinte freqüência: doença de membrana hialina $(21,5 \%)$; infecções intra-uterinas (20,5\%); asfixia (18,2\%); malformações $(15,6 \%)$ e infecções pós-natais bacterianas $(8,6 \%)^{7}$. No hospital da Asa Sul de Brasília (89-91), as principais causas de morte neonatal foram a asfixia (32\%), as infecções (30\%), seguidas da doença de membrana hialina (20\%), e as malformações fatais $(14 \%)^{8}$. Os dados de Toronto (80-84), Quebec (80-82) e Carolina do Norte (80-84) mostraram as malformações fatais e prematuridade extrema como as principais causas. Essas duas enfermidades são consideradas, usualmente, como patologias não-preveníveis, e as demais refletem deficiências de assi stência médica no pré-natal eno parto das gestantes e no recém-nascido, o mau atendimento na sala de parto e na terapia intensiva neonatal ${ }^{5,11,14-17}$.

A análise dos natimortos mostrou como primeira causa a asfixia perinatal, em geral, representando as dificuldades de assistência ao parto, pois nem sempre é detectada. Infecções intra-uterinas vêm em segundo lugar e estão relacionadas com as infecções maternas não-tratadas; muitas vezes de difícil diagnóstico, como a relação entre bolsa rota e infecção. I sso é mais grave na prematuridade, em que, muitas vezes, a infecção é a causa do parto prematuro, além da sua deficiência imunológica11,15,17. Assim, somente o controle adequado da gestante no pré-natal asseguraria a detecção e o tratamento adequado da infecção intraparto ${ }^{5,10}$. Nos estudos norte-americanos, os seguintes fatores de risco são significativos: gestantes de cor não-branca, sol teiras, adolescentes, drogaditas e sem pré-natal. I sso representa populações de baixa renda, cujas más condições socioeconômicas transcendem fatores diretamente relacionados à saúde. Entretanto, é necessário que a rede de assistência médica, pública e privada, seja integrada e hierarquizada, possibilitando redução gradual na mortalidade materna, fetal e neonatal. A asfixia, a infecção e a doença da membrana hialina são enfermidades potencialmente preveníveis. Restariam as enfermidades ainda não passíveis de prevenção, como as malformações graves e a prematuridade extrema de menores de 750 gramas, ao nascer ${ }^{9,13,15}$.

\section{CONCLUSÕES}

Os avanços tecnológi cos e a capacitação do pessoal médico e de enfermagem, nos cuidados intensivos neonatais, não provocaram redução nos coeficientes de mortalidade neonatal. A penas uma tendência ao declínio no grupo de RN menores de $1.500 \mathrm{~g}$. Houve aumento nas causas de morte por infecções pós-natais ou hospitalares e nas malformações congênitas graves.

\section{SUMMARY}

\section{Perinatal and neonatal mortality at the Hospi- tal de Clínicas de Porto Alegre, Brazil}

Objective - Epidemiological analysis of neonatal and perinatal mortality of 20,280 newborns alive with $500 \mathrm{~g}$ or more and 374 stillbirths occurred at the Hospital deClínicas dePorto Al egrefrom 1984 to 1990 .

Purpose - To compare two periods: A (19841987) with B (1988-1990), estabilishing a relationship between the changes occurred in the causes and the rate of mortality.

Methods - The retrospective study was done with the records of promptuaries of obstetrical and neonatal centers, and review of flow-sheets of the deaths and autopsies.

Results - Between 1984 to 1990, 20,280 newborns alive with $500 \mathrm{~g}$ or more, 374 stillbirths at perinatal unit of Hospital de Clínicas de Porto Alegre were born. 258 deaths occurred, the neonatal mortality rate was 12.7 per thousand. The stillbirth rate was 18.4 per thousand. The perinatal mortality rate was 28.4 per thousand. The incidence of I ow birth weight $(<2,500 \mathrm{~g})$ was $11,2 \%$ and very low birth weight $(<1,500 \mathrm{~g})$ was $1.8 \%$, the former group had an increase incidence between 1984-1988 (A) from $1.5 \%$ to $2.2 \%$ (B). The causes of deaths were distributed as follow: a) intrauterine infections (22.4\%); b) hyaline membrane disease (20.1\%); c) congenital malformation (18.2\%); d) asphyxia (15.5\%); e) postnatal infections (9.7\%). The causes of stillbirth were: a) perinatal asphyxia $(38.7 \%) ;$ b) intrauterine infections $(9 \%)$; toxemia $(8.2 \%)$; d) malformation (7.4\%). The period B showed changes with an increase of postnatal infections odds ratio (OR) 7 (1.9-30.6) and congenital malformations OR 1.6 (0.8-3.2). It did not occurred a decrease in mortality rate for prematures bel ow 1,500g OR 90 (61-118) in A to 54 (37-68) in B.

Concl usions - The advances in technology and human capacity were not sufficient to reduce significantly the rate of neonatal mortality. [Rev Ass Med Brasil 1997; 43(1): 35-9.]

KEY WORDS: Newborn. Perinatal mortality.

\section{REFERÊNCIAS BIBLIOGRÁFICAS}

1. Mortalidade Infantil em PortoAlegre. Bol etim I nformativo da Sociedade de Pediatria do Rio Grande do Sul, 1992; 36: 4.

2. Dollfus C, Patteta M, Siegel E, Cross AW. Infant mortality: a practical approach to the analysis of the leading causes of death and risk factors. Pediatrics 1990; 86: 176-83.

3. Miura E. Causas demortalidadeperinatal. In Miura E : Neona- 
tologia, Princípios ePrática. Porto Alegre, Ed Artes Médicas, 1 a ed, 1991; 7-8.

4. Ehrenhaft $P M$, Wagner J L, Herdman RC. Changing prognosis for very low birth weight infants. Obstetr Gynecol 1989; 74: 528-35.

5. Guide for the study of perinatal mortality. Quebec Perinatal Committee, Corporation of Physicians of Quebec, Montreal, Canadá, 3rd ed,1982.

6. NHDS, National Hospital DischargeSurvey: casefatality rates associated with conditions originating in the perinatal period: United States, 1986 through 1987. Pediatrics 1992; 89: 1.256-9.

7. Ohlson A, Shennan AT, RoseTH. Review of causes of perinatal mortality in a regional perinatal center, 1980 to 1984. Am J Obstet Gynecol 1987; 157: 443-5.

8. Margotto PR. Mortalidade perinatal, Unidade de Neonatologia HRAS/1991. Bol etim I nformativo Pediátrico. Brasília, 1992; 55: 2-22.

9. Fiori RM, Fiori $\mathrm{HH}$, Hentschel $\mathrm{H}$. Mortalidade perinatal no Rio Grande do Sul. J Pediatr 1989; 65: 72-85.

10. Hein HA, Lathrop SS. The changing pattern of neonatal mortality in a regionalized system of perinatal care. AJ DC 1986; 140: 989-93.
11. Phelps DL, Brown DR, Tung B et al. 28 day survival rates of 6676 neonates with birth weights of 1250 grams or less. Pediatrics 1991; 87: 7-17.

12. Souto A. A sobrevida dos prematuros de muito baixo peso. Anais do XXVII Congresso Brasileiro de Pediatria. Porto Alegre, 1991; 156.

13. Bailey CF, Katwinkel J, Veith ST, Fergunson E. A study of preventable vs non-preventable causes of neonatal mortality. Pediatr Res (abstracts of APS-SPR) 1992; 31(4): 242A.

14. Krishnan V, Fritz D. Regionwideanalysis of neonatal mortality in Northest Ohio. Pediatr Res (abstract of APS-SPR) 1992; 31: $253 \mathrm{~A}$

15. Telsey AM, Kandall SR, Damus K. Reversal of infants and neonatal mortality rates for low birth weight whites and nonwhites in N ew York city. Pediatr Res. (abstracts of APS-SPR) 1991; 30: 268A.

16. Lofgren O, Polberger S. Perinatal mortality: changes in the diagnostic panorama 1974-1980. Acta Paediatr Scand 1983; 72: 27-32.

17. Autio-Harmainen H, Rapola J , Hoppu K, Osterlund K. Causes of neonatal deaths in a Pediatric H ospital N eonatal U nit. Acta Paediatr Scand 1983; 72: 333-7. 